AperTO - Archivio Istituzionale Open Access dell'Università di Torino

\title{
Probing lactate secretion in tumours with hyperpolarised NMR
}

\section{This is a pre print version of the following article:}

Original Citation:

Availability:

This version is available http://hdl.handle.net/2318/1619399

since 2016-11-30T23:24:33Z

Published version:

DOI:10.1002/nbm.3574

Terms of use:

Open Access

Anyone can freely access the full text of works made available as "Open Access". Works made available under a Creative Commons license can be used according to the terms and conditions of said license. Use of all other works requires consent of the right holder (author or publisher) if not exempted from copyright protection by the applicable law. 


\section{Probing Lactate Secretion in Tumours with Hyperpolarised Nuclear Magnetic Resonance}

Markus Durst ${ }^{1,2}$, Ulrich Koellisch ${ }^{1,2}$, Valeria Daniele ${ }^{3}$, Katja Steiger ${ }^{5}$, Markus Schwaiger ${ }^{5}$, Axel Haase $^{1}$, Marion I. Menzel ${ }^{2}$, Rolf F. Schulte ${ }^{2}$, Silvio Aime ${ }^{3}$, Francesca Reineri ${ }^{3}$.

${ }^{1}$ IMETUM, Technische Universität München, Garching, Germany

${ }^{2}$ GE Global Research, Garching, Germany

${ }^{3}$ University of Torino, Turin, Italy

${ }^{5}$ Nuklearmedizinische Klinik und Poliklinik, Klinikum rechts der Isar, Technische Universität München, München, Germany

\section{Corresponding author:}

Markus Durst

Technische Universität München

Institute of Medical Engineering

Boltzmannstraße 11

85748 Garching b. München

Germany

Phone: +4989-289-10841

Fax: $\quad+4989-289-10805$

Email: markus.durst@tum.de

Short running title:

Probing Lactate Secretion in Tumours with Hyperpolarised NMR

Keywords:

Hyperpolarized C13, Gd chelate based contrast agents, Spectroscopic imaging, Breast cancer Abbreviations:

FID free induction decay; DNP dynamic nuclear polarization; NUFFT non-uniform fast Fourier transform; MCT monocarboxylate transporter; FDG fluorodeoxyglucose; PET positron emission tomography; LDH lactate dehydrogenase; ALT alanine transaminase; ECG Electrocardiography; FRFSE fast-recovery fast spin-echo; DCE dynamic contrast-enhanced

Word Count: 6542 


\section{Abstract}

Most tumours exhibit a high rate of glycolysis and predominantly produce energy by lactic acid fermentation. To maintain energy production and prevent toxicity, the lactate generated needs to be rapidly transported out of the cell. This is achieved by monocarboxylate transporters (MCTs), which therefore play an essential role in cancer metabolism and development.

In vivo experiments were performed on 8 male Fisher F344 rats bearing a subcutaneous mammary carcinoma after injection of hyperpolarised [1-13C]pyruvate. A Gd(III)DO3A complex that binds to pyruvate and its metabolites was used to efficiently destroy the extracellular magnetisation after hyperpolarised lactate had been formed. Moreover, a pulse sequence including a frequency selective saturation pulse was designed so that the pyruvate magnetisation could be destroyed to exclude effects arising from further conversion. Given this preparation, metabolite transport out of the cell manifested as additional decay and apparent cell membrane transporter rates could thus be obtained using a reference measurement without a relaxation agent. In addition to slice-selective spectra, spatially resolved maps of apparent membrane transporter activity were acquired using a single-shot spiral gradient readout.

A considerable increase in decay rate was detected for lactate, indicating rapid transport out of the cell. The alanine signal was unaltered which corresponds to a slower efflux rate.

This technique could allow for better understanding of tumour metabolism and progression; and enable treatment response measurements for MCT-targeted cancer therapies. Moreover, it provides vital insights into the signal kinetics of hyperpolarised [1-13C]pyruvate examinations. 


\section{Introduction}

In contrast to normal cells, most cancer cells exhibit a high rate of glycolysis and predominantly produce energy by fermenting the generated pyruvate to lactate even in the presence of oxygen $(1,2)$. This phenomenon, also called the Warburg effect, is the foundation for FDG PET examinations routinely used in clinical practice for diagnosis and treatment response monitoring in cancer patients.

To maintain energy production and prevent toxicity, the generated lactate needs to be rapidly transported out of the cell. Pyruvate and lactate cannot cross the cell membrane by free diffusion due to their electric charge but their transport is catalysed by proton-linked monocarboxylate transporters (MCTs) (3). These transporter proteins are driven solely by the concentration gradients of the target molecule and surrounding protons, thereby also contributing to regulation of intra- and extracellular $\mathrm{pH}$.

Excessive lactate production and secretion are linked to many crucial steps in the progress of cancer: High levels of lactate in tumours are related to increased metastases and cancer cell resistance to radiotherapy (4-6) as well as to significantly lower overall survival rates of patients (7-9). Studies indicate that exogeneous lactate stimulates tumour angiogenesis, growth and metastasation (10-14). Moreover, high extracellular lactate concentrations were found to reduce the immune response to cancer cells by compromising the metabolism of immune cells (15-17). There is also a symbiotic relationship between cancer cells which release lactate as a waste product and other cancer cells which use it to fuel their energy production. Overall, MCTs play an essential role in cancer metabolism and development (1820). Therefore, a deeper understanding of MCT activity in tumours is highly desired and is currently the subject of intense research.

As MCTs occupy a key position in the energy production of cancer cells, they also represent a promising target for new cancer therapies (21). For example, $\alpha$-cyano-4-hydroxycinnamic acid, a drug that inhibits lactate transport in mammalian cells, was shown to reduce growth and induce necrosis in cancer cells by restricting lactate efflux thus impairing glycolytic energy production (22-25).

Recently, dynamic nuclear polarisation (DNP) with subsequent dissolution (26) has enabled monitoring of uptake and metabolism of endogenous biomarkers by nuclear magnetic resonance methods (27-29). Dissolution DNP can achieve a signal increase of 10.000-fold or more for a pyruvate solution which can be used to study pyruvate-to-lactate conversion in tumours (30-33). However, the measured lactate signal not only reflects enzymatic conversion but is also affected by various other parameters such as perfusion, cell membrane transport, intra- and extracellular pool sizes and polarisation decay. Various approaches have been introduced to disentangle this complex interplay of biological processes such as direct quantification of inflow and outflow in bioreactors $(34,35)$, using saturation and inversion transfer methods to quantify the label exchange between substrate and metabolite pools (36), injecting hyperpolarised alanine to determine intracellular 
pyruvate and lactate levels (37), using commercial relaxation agents that partially destroy extracellular magnetisation to measure altered conversion rates (38), and exploiting different $T 2$ relaxation $(39,40)$ or using diffusion gradients $(41-45)$ to distinguish different microenvironments. However, these methods suffer from serious drawbacks involving challenges for in vivo applicability, lack of sensitivity, or ambiguity of results. Currently, a method for directly measuring MCT activity in vivo is not available.

Here, we use a Gd(III)DO3A complex (46) that binds to pyruvate and its metabolites to efficiently destroy extracellular magnetisation after hyperpolarised lactate has been formed. Both slice-selective spectroscopic and imaging data were acquired in rats with a subcutaneous mammary carcinoma. In combination with a newly developed pulse sequence that saturates pyruvate magnetisation, we measured considerably increased lactate signal relaxation in the presence of $\mathrm{GdDO} 3 \mathrm{~A}$ which indicated high rates of lactate efflux from the tumour cells.

\section{Theory}

\section{GdDO3A}

The idea of quenching the extracellular biomarker hyperpolarisation has been reported earlier $(38,47)$; however, commercially available relaxation agents can only provide limited relaxation since they rely solely on outer-sphere interaction with the hyperpolarised molecule. In this study, we found that much higher efficiency in destroying the hyperpolarisation could be achieved using a coordinatively unsaturated Gd(III)DO3A complex (gadolinium-1,4,7,10-tetraazacyclododecane-1,4,7-triacetic acid). The heptadentate ligand leaves two vacancies in the inner coordination sphere of the $\mathrm{Gd}(\mathrm{III})$ ions that can be occupied by donor atoms from pyruvate or its metabolites. For several $\alpha$-hydroxycarboxylates such as lactate, the formation of a stable ternary adduct has been observed (46) as shown in Figure 1. More information about this Gadolinium-chelate and its properties can be found in $(46,48)$. Therefore, it is expected that GdDO3A will provide increased relaxation compared to coordinatively saturated complexes such as Gd(HP-DO3A) or Gd(BMADTPA) for these compounds. Due to the size of the complex, it will remain outside of the cell and can therefore be used to specifically quench extracellular magnetisation.

\section{In vivo pyruvate signal kinetics}

After injection, the hyperpolarised pyruvate arrives at the tumour site where it is taken up by the cancer cells via MCTs. Inside the cell, pyruvate is metabolised mainly to lactate by lactate dehydrogenase (LDH) and to alanine by alanine transaminase (ALT). The lactate generated is then exported to the extracellular space again by MCTs. To deduce the transporter activity, pyruvate signal kinetics need to be described by a multicompartment model considering intra- and extracellular signal portions. Assuming constant conversion and transporter rates, and neglecting back reaction and back transport, this will result in an intricate set of coupled differential equations: 


$$
\begin{aligned}
& \frac{d}{d t} P_{\text {ext }}=-R_{1, \text { ext }} P_{\text {ext }}-k_{P, \text { ext } \rightarrow \text { int }} P_{\text {ext }}+r_{i f} \\
& \frac{d}{d t} P_{\text {int }}=-R_{1, \text { int }} P_{\text {int }}+k_{P, \text { ext } \rightarrow \text { int }} P_{\text {ext }}-k_{P \rightarrow L} P_{\text {int }}-k_{P \rightarrow A} P_{\text {int }} \\
& \frac{d}{d t} L_{\text {ext }}=-R_{1, \text { ext }} L_{\text {ext }}+k_{L, \text { int } \rightarrow \text { ext }} L_{\text {int }} \\
& \frac{d}{d t} L_{\text {int }}=-R_{1, \text { int }} L_{\text {int }}+k_{P \rightarrow L} P_{\text {int }}-k_{L, \text { int } \rightarrow \text { ext }} L_{\text {int }}
\end{aligned}
$$

where $\mathrm{P}$ and $\mathrm{L}$ are the longitudinal pyruvate and lactate magnetisations, $k_{P \rightarrow L}$ and $k_{P \rightarrow A}$ are the apparent conversion rates for pyruvate-to-lactate and pyruvate-to-alanine conversion, $k_{P, \text { ext } \rightarrow \text { int }}$ and $k_{L, \text { int } \rightarrow \text { ext }}$ are the apparent rates for cell membrane transport of pyruvate into and of lactate out of the cell, $r_{i f}$ is the net pyruvate inflow rate, $R_{1}$ is the spin-lattice relaxation rate and the indices ext and int denote the extra- and intracellular compartments, respectively.

A standard FID signal does not contain any information on compartmentalisation and is the total of intra- and extracellular components. To crush the extracellular magnetisation, GdDO3A can be injected when lactate is formed and the results of this measurement can be compared to those of an acquisition without the relaxation agent. However, the ongoing inflow, uptake and conversion of pyruvate results in a different lactate signal modulation since pyruvate will also be crushed when GdDO3A is present. Furthermore, the lactate signal in the measurement with GdDO3A can still be modulated due to the conversion of intracellular pyruvate and label exchange from alanine over pyruvate to lactate. To remove these effects, a frequency selective saturation pulse on pyruvate can be used to quench the pyruvate magnetisation in both measurements, leaving only the magnetisation of the metabolites. Then, the lactate magnetisation can be described by two simplified coupled differential equations for extra- and intracellular magnetisation:

$$
\begin{aligned}
& \frac{d}{d t} L_{\text {ext }}=-R_{1, \text { ext }} L_{\text {ext }}+k_{L, i n t \rightarrow e x t} L_{i n t} \\
& \frac{d}{d t} L_{\text {int }}=-R_{1, i n t} L_{\text {int }}-k_{L, \text { int } \rightarrow \text { ext }} L_{\text {int }}
\end{aligned}
$$

where, for the measurement with GdDO3A, $R_{1, \text { ext }}$ is time-dependent due to the inflowing contrast agent.

When GdDO3A is injected in high concentrations and a waiting time is implemented between contrast agent injection and data acquisition, a high extracellular concentration of 
GdDO3A can be assumed. Therefore, the lactate transported to the extracellular compartment will be crushed almost immediately and the signal equation simplifies again to

$\frac{d}{d t} L_{i n t}=-R_{1, i n t} L_{i n t}-k_{L, i n t \rightarrow e x t} L_{i n t}$

The apparent membrane transporter rate can then be calculated straight forward as the difference in the measured decay rates between the acquisition with and without added GdDO3A:

$k_{L, \text { int } \rightarrow \text { ext }}=R_{L, e f f, G d}-R_{L, e f f}$

where $R_{L, e f f, G d}$ denotes the effective relaxation rate of lactate with added GdDO3A and $R_{L, e f f}$ the effective relaxation rate of lactate without GdDO3A. The behaviour of alanine magnetisation is analogous to that of lactate magnetisation. Please see also Figure 2 for a graphical illustration.

\section{Materials and Methods}

\section{GdDO3A relaxation properties}

The longitudinal relaxation times of the $13 \mathrm{C}$ label of the three substrates [1-13C] pyruvate, [1-13C] lactate and [1-13C] alanine with different ratios of GdDO3A were quantified in serum (FBS). The concentration of metabolites and of the paramagnetic contrast agent was similar to the one obtained in in vivo experiments $(5 \mathrm{mM}$ pyruvate concentration, assuming a $1 \mathrm{ml}$ injection of $80 \mathrm{mM}$ pyruvate is diluted in $15 \mathrm{ml}$ of rat blood). The effect of the GdDO3A complex was compared with that of the coordinatively saturated Gd(HP-DO3A) (trade name: ProHance). These measurements were performed at $14 \mathrm{~T}$ (Bruker Avance $600 \mathrm{MHz}$ spectrometer), using the inversion recovery pulse sequence.

\section{Hyperpolarisation}

[1-13C]pyruvic acid doped with $15 \mathrm{mM}$ trityl OX063 radical and $1 \mathrm{mM}$ Dotarem was polarised in a HyperSense DNP polariser (Oxford Instruments, Abingdon, UK). After $1 \mathrm{~h}$, the frozen sample was rapidly dissolved by flushing with a hot aqueous solution containing 80 $\mathrm{mM}$ Trizma buffer, $80 \mathrm{mM} \mathrm{NaOH}$, and $0.1 \mathrm{~g} / \mathrm{L} \mathrm{Na-EDTA}$, resulting in an $80 \mathrm{mM}$ pyruvate solution with physiological $\mathrm{pH}$, osmolarity, and temperature. The liquid-state polarisation was approximately $25 \%$.

\section{Scanner setup}

In vivo experiments were performed on a clinical 3T HDx scanner (GE Healthcare, Milwaukee, $\mathrm{WI}$ ) with maximum gradient amplitude of $40 \mathrm{mT} / \mathrm{m}$ and maximum slew rate of $150 \mathrm{~T} / \mathrm{m} / \mathrm{s}$. A dual-tuned 13C-1H birdcage volume coil (diameter: $8 \mathrm{~cm}$ ) was used for radiofrequency transmission and reception (49).

\section{Animal handling}


The proposed protocol was tested in 8 male Fisher F344 rats (Charles River) with a subcutaneous Mat BIII tumour in the neck region. Mat BIII syngeneic cells from rat mammary adenocarcinoma were maintained in McCoy's 5a Medium Modified (catalogue no. 30-2007; ATCC, Manassas, MA) supplemented with $10 \%$ FCS. Cells were kept in a humidified atmosphere at $5 \% \mathrm{CO} 2$ and $37^{\circ} \mathrm{C}$. At the time of implantation they were harvested and washed twice; approximately $10^{6}$ cells were implanted subcutaneously into the shoulder in serum-free medium. Tumours reached a size of $\sim 1.5 \mathrm{~cm}$ within 10 days after implantation.

During the experiment, the animals were anaesthetised with 1-3\% isoflurane gas inhalation. They were monitored using ECG and for breathing, oxygen saturation and temperature, and were kept warm on a heating pad with circulating warm water. Then, $1 \mathrm{ml}$ of dissolved 80 $\mathrm{mM}$ hyperpolarised pyruvate solution was injected into the tail vein. The animal study was approved by the local governmental committee for animal protection and welfare (Tierschutzbehörde, Regierung von Oberbayern).

\section{Pulse sequence design}

A 2D spectral-spatial RF pulse to selectively excite pyruvate or its metabolites was designed by direct inversion of the excitation matrix $(50,51)$. The pulse consisted of 18 lobes with total pulse length of $22.4 \mathrm{~ms}$ and used flyback-gradients to increase robustness against gradient errors.

For spatial encoding, a single-shot spiral gradient readout (FOV $8 \mathrm{~cm}$; resolution $24 \times 24$ pixels; maximum gradient amplitude $13 \mathrm{mT} / \mathrm{m}$; maximum gradient slew rate $46 \mathrm{~T} / \mathrm{m} / \mathrm{s}$ ) was created using the variable density spiral generation script by Brian Hargreaves (52).

A spectroscopic acquisition sequence was implemented using the spectral-spatial RF pulse. First, the pulse was executed on the pyruvate frequency without slice selection using a flip angle of $90^{\circ}$ and a subsequent spoiler gradient. Then, lactate and alanine signal was acquired slice-selectively with a flip angle of $6^{\circ}$ in subsequent excitations with a repetition time of 100 ms so that a full set of metabolite signals with preceding pyruvate saturation was obtained every $300 \mathrm{~ms}$. The readout time was $51.2 \mathrm{~ms}$ with a readout bandwidth of $5 \mathrm{kHz}$ and a total of 256 points.

For imaging, the repetition time was increased to $200 \mathrm{~ms}$ and the pulse sequence was modified to include three excitations using the pyruvate saturation pulse without slice selection followed by slice-selective excitations with $20^{\circ}$ on the lactate and alanine frequency. The single-shot spiral readout gradient was used for spatial encoding. A full set of metabolite images with preceding pyruvate saturation could be obtained every second. The readout time was $65.5 \mathrm{~ms}$ with a readout bandwidth of $62.5 \mathrm{kHz}$ and a total of 4096 points. For a schematic drawing of the individual pulse sequences, please see Figure 3.

\section{Measurement protocol}


For each animal, two separate injections were performed $1 \mathrm{~h}$ apart: the first without and the second with administration of $0.5 \mathrm{ml}$ of a GdDO3A solution $16 \mathrm{~s}$ after the pyruvate injection at the supposed plateau of the lactate signal. The relative amount of GdDO3A compared to pyruvate was chosen to be 1:5 for 7 rats and 1:1 for one rat to test concentration effects. After $20 \mathrm{~s}$, the pulse sequence described above was started. Spectroscopic data were acquired for 4 rats and imaging data for another 4 rats in a 14-18 $\mathrm{mm}$ slice including the tumour. In the time between the two injections, fast-recovery fast spin-echo (FRFSE) $1 \mathrm{H}$ images with slice thickness of $3 \mathrm{~mm}$ were acquired in the centre of the $13 \mathrm{C}$ tumour slice to provide anatomical reference.

\section{DCE study}

The time dependence of the concentration of the paramagnetic complex in the tumour tissue during the first few seconds after the injection has been separately studied at a 7T small animal scanner (Agilent Technologies) using four rats bearing a Mat BIII tumour. A time resolved imaging of contrast kinetics (TRICKS) sequence was used to acquire T1-weighted proton images every $2.67 \mathrm{~s}$ (resolution $128 \times 128$ pixels, FOV $8 \mathrm{~cm}$ ). The injection of GdDO3A contrast agent followed the same protocol as used for the hyperpolarised acquisitions. Relaxation rates for extended modelling were calculated based on the in vitro results by assuming a homogeneous distribution of contrast agent in the rat blood volume.

\section{Immunohistochemistry}

Separately implanted subcutaneous MAT BIII tumours were fixed in $4 \%$ neutral-buffered formalin, routinely embedded in paraffin and cut into $2 \mu \mathrm{m}$ thick consecutive slices. Immunohistochemistry to detect MCT 1 (Merck-Millipore AB1286-I) and MCT 4 (Santa Cruz Biotechnologies sc-50329) expression was performed after heat-mediated antigen retrieval for 20 minutes in citrate buffer ( $\mathrm{pH} 6)$ with goat anti-chicken (MCT1, medac diagnostics 1624-06) or goat anti-rabbit (MCT4, medac diagnostics 71-00-30) secondary antibodies and a strepatvidine-peroxidase detection system (medac diagnostics 71-00-38) visualized by Dab (medac diagnostics BSO4).

\section{Reconstruction}

A $15 \mathrm{~Hz}$ Gaussian filter was applied along the readout dimension to both spectroscopy and imaging data. Fast Fourier transform (FFT) was applied to the spectroscopy data to reconstruct spectra. Spiral imaging data were phase corrected along the readout for the resonance frequency of the specific metabolite. Subsequently, a non-uniform fast Fourier transform (NUFFT) (53) was used to reconstruct spiral data onto a Cartesian grid.

Since in vivo acquisitions of hyperpolarised biomarkers typically suffer from low SNR compared to standard proton acquisitions, it is important to avoid the Rician bias (54) from absolute value images or spectra when fitting the decay rates. Thus, the spectra were phase corrected and the peak value of the real valued spectrum was used for further evaluation. In this special case, the images feature the same structures with decreasing SNR; thus the 
mean phase from the first few images with sufficient SNR (where the noise is nearly Gaussian distributed) was taken to correct the whole image series. For the lactate imaging acquisitions, regions of interest (ROIs) were drawn manually to calculate the mean tumour signal. As alanine is not specific to the tumour tissue, ROls were generated by taking each image voxel with a signal $>70 \%$ of the maximum signal within the first image.

A monoexponential fit was applied to the decay curves of the metabolites starting $3 \mathrm{~s}$ after beginning the acquisition when pyruvate was completely saturated and the signal dynamics had reached equilibrium. Apparent cell transporter activity rates were calculated according to equation (8). Furthermore, spatially resolved maps of the apparent lactate MCT transporter rate were obtained by applying the fit on each voxel with a signal $>30 \%$ of the maximum signal within the first image.

To judge the validity of the assumption that the extracellular compartment is crushed immediately, the spectroscopic data that had a higher time resolution were fitted from the start using equations (5) and (6) with the time-dependent $\mathrm{R} 1$ relaxation values derived from proton DCE measurements.

\section{Results}

\section{GdDO3A relaxation properties}

The $T_{1}$ values measured in vitro are reported in Table 1 . Compared to the coordinatively saturated Gd(HP-DO3A) complex (trade name: ProHance), GdDO3A could achieve an approximately 10 -fold reduction of $\mathrm{T}_{1}$ relaxation times for lactate and alanine in serum. It must be mentioned that the in vitro measurements were carried out at $14 \mathrm{~T}$, while the in vivo experiments are made at 3T. However, while the $\mathrm{T} 1$ of the ${ }^{13} \mathrm{C}$ carboxylate metabolites' signal is strongly dependent on the magnetic field strength, the paramagnetic contribution of the Gd-DO3A to the relaxation rate is the same in the two different fields.

\section{DCE study}

Consistent with literature reports of commercial Gd-complexes, it was found that immediately after the injection of the GdDO3A contrast agent in the vein, its concentration in the tumour area increases in the first 20 seconds, after which it is slowly washed out. The time dependent relaxation rates (R1) of the metabolites were calculated and are shown in Figure 4. The measurements confirm that a sufficient concentration of GdDO3A is reached during the hyperpolarised $13 \mathrm{C}$ acquisition. Furthermore, from previous reports of contrast agents of similar molecular size (55), it is known that the bulk part of the contrast agent is directly excreted into the interstitial space. The signal time evolution is shown in Supplementary Figure 1, and the derived relaxation curves are given in Supplementary Figure 2.

\section{Immunohistochemistry}


Tumours of subcutaneous Mat BIII syngrafts show a strong membranous MCT1 and a moderate membraneous MCT4 expression (see Supplementary Figures 3 and 4).

\section{In vivo hyperpolarisation experiments}

The fitted apparent efflux rates of lactate $\left(k_{L, \text { int } \rightarrow \text { ext }}\right)$ and alanine $\left(k_{A, \text { int } \rightarrow \text { ext }}\right)$ can be found in table 2 . The mean value of $k_{L, \text { int } \rightarrow \text { ext }}$ from the spectroscopy acquisitions (rat 1-3) was $0.20 \pm$ $0.05 \mathrm{~s}^{-1}$, the mean value from the imaging acquisitions (rat 5-8) $0.18 \pm 0.03 \mathrm{~s}^{-1}$. For alanine, no significant difference in decay rates was found within the noise level (spectroscopy rat 13: $0.01 \pm 0.01 \mathrm{~s}^{-1}$, imaging rat 5-8: $0.02 \pm 0.02 \mathrm{~s}^{-1}$ ). Rat 4 with a pyruvate to $\mathrm{Gd}$ ratio of 1:1 shows a slightly elevated apparent export rate for lactate $\left(0.31 \mathrm{~s}^{-1}\right)$ whereas the alanine signal is unchanged again. To verify the effectiveness of the pyruvate saturation pulse, the pyruvate spectroscopy signal was analysed for the first injection without relaxation agent. The remaining pyruvate magnetisation in the entire rat body was found to be $4 \% \pm 5 \%$ at the beginning of the decay rate fit. The overlays of the $13 \mathrm{C}$ metabolite maps with the anatomical proton images confirmed that most of the lactate signal in the slice stemmed from the tumour, whereas alanine was distributed mostly in the surrounding tissue (data not shown). The SNR of the lactate images was sufficient to derive spatially resolved maps of $k_{L, \text { int } \rightarrow \text { ext }}$ which showed homogeneous apparent transporter activity within the tumour area. Exemplary datasets for spectroscopy and imaging are presented in Figures 5 and 6 , respectively.

The extended model including extra- and intracellular compartments with a time-dependent extracellular relaxation rate yielded slightly increased values for $k_{L, \text { int } \rightarrow \text { ext }}$ as compared to the simple decay rate fitting model ( $16 \%$ increase on average). For $k_{A, \text { int } \rightarrow \text { ext }}$, the results were unchanged.

\section{Discussion and Conclusions}

From relaxation rate measurements carried out in vitro (in plasma) it is clear that the GdDO3A complex allows extremely fast relaxation of the signal of hyperpolarised metabolites, much faster than using other commercial Gd(III) contrast agents, even when the concentration of both the paramagnetic complex and the metabolite is lower than $1 \mathrm{mM}$. Judging from the in vitro results, GdDO3A is therefore a superior candidate to create contrast between intra- and extracellular magnetisation for hyperpolarised pyruvate experiments. Furthermore, in a separate DCE study we found that the GdDO3A concentration rises quickly in the tumour during the hyperpolarised $13 \mathrm{C}$ acquisition. However, a quantitative measurement as well as a comparison with commercially available contrast agents in a future in vivo study might be advisable.

In vivo data from a preliminary study with GdDO3A conducted in healthy rats are shown in Figure 4. Significant alteration of signal kinetics can be observed for both lactate and alanine, which could be due to efflux from the cells or loss of hyperpolarised flux from pyruvate. Using standard NMR methods, this dilemma cannot be resolved as there are too many 
unknowns to fit the curves to the required 6 compartment model. Even if more information was available, the fit would most likely be error-prone due to the typically low SNR regime of hyperpolarised acquisitions. The proposed method elegantly reduces the problem to simply fitting the decay rates by completely eliminating the influence of pyruvate conversion.

We found a significant difference of lactate decay in the presence of GdDO3A, which indicates considerable lactate efflux in the tumour tissue. This is also consistent with the immunohistochemical analysis, which shows a strong membranous MCT1 expression and a moderate membranous MCT4 expression. These are two of the most important transporters responsible for lactate transport. While MCT4 is the transporter believed to be mainly responsible for the export of glycolysis-derived lactate occurring in hypoxic tumours $(56,57)$, there is evidence that under certain circumstances, also MCT1 can become a major pathway for lactate efflux (13).

For alanine, no significant difference in decay rates could be found, suggesting that alanine excretion occurs on a much slower time scale. This is consistent with our assumptions, since alanine is an amino acid and not transported by MCTs but by a range of other transporter systems that require the co-transport of other amino acids (58). As a non-essential aminoacid, alanine mostly serves as an exchange substrate for the uptake of essential amino acids in mammalian cells. We therefore expected its efflux rate to be at least one order of magnitude lower than the values found for lactate (59). These results confirm the sensitivity of the method for detecting lactate transport out of the cell.

The results obtained from the extended model are slightly higher than those for the simple model which assumes instantaneous quenching of the extracellular compartment. This was expected, since a lower relaxation rate in the extracellular compartment translates to higher actual transporter rates. This is also indicated in rat 3 where a higher GdDO3A concentration was used, and the values from both models were similar. Therefore, results of the extended model confirm that the assumption of immediate relaxation in the extracellular compartment is practicable and will introduce only little error regarding influence of the inflowing contrast agent. This simple model also avoids the necessity of acquiring DCE proton data. To improve accuracy, future studies should use a higher concentration of GdDO3A if possible.

As there is no gold standard to compare the results to, the limitations of the described technique must be thoroughly evaluated in further studies. In principle, the same challenges arise as for the apparent conversion rate constants describing the metabolic conversion. As a result of relaxation, the hyperpolarised in vivo signal cannot be directly related to absolute molecule concentrations. Also, the measurements may be complicated by underlying biological processes such as perfusion, label exchange and other influences. One important factor is the perfusion and accumulation of the GdDO3A agent; results may be biased by insufficient concentration or slow inflow into the target tissue. This is indicated by rat 4 , which received a higher concentration of the GdDO3A compound, resulting in elevated values of the decay. If quantitative proton T1 maps and $13 \mathrm{C}$ data can be acquired in the 
same scan, as demonstrated in (38), the obtained concentration of GdDO3A may be used to improve accuracy and provide more information on the dynamics during the GdDO3A bolus. To increase the relaxation weighting, a higher concentration of GdDO3A should be used in future examinations if possible. Moreover, a real-time bolus tracking approach could be adapted to automatically detect the optimal time point for starting the acquisition at the peak of the lactate curve (60). Furthermore, the influence of the intra- and extracellular pool sizes needs to be investigated. If there is a large extracellular pool, most of the signal may stem from the decay of this pool. Also, it is currently not possible to distinguish between net transport and label exchange between the intra- and extracellular pool. In (44), diffusionsensitive measurements imply that the vascular fraction of pyruvate and lactate is rather small in prostate tumours. Regarding this issue, the pulse sequence could be modified to acquire spin echoes. This may allow better investigation of the extra- and intracellular compartment sizes of the metabolite signal due to varying $\mathrm{T} 2$ relaxation time with and without GdDO3A administration. Another interesting approach could be to incorporate the GdDO3A complex into a liposome structure to target only intravascular hyperpolarisation. This may provide valuable complementary information on the distribution of metabolites between vascular, interstitial, and intracellular spaces. For these reasons, tumour models with a different rate of lactate export have to be studied in order to determine whether the contrast provided by the proposed method is sufficient to distinguish between different tumour types or disease states.

In conclusion, we present a method that potentially allows direct measurement of cell membrane transport in vivo using hyperpolarised biomarkers. Significantly increased decay rates were found for lactate in rats with mammary carcinoma in the presence of GdDO3A, indicating rapid transport out of the cell. In addition to slice-selective spectra, spatially resolved maps of apparent membrane transporter activity could be obtained. This technique could allow for better understanding of tumour metabolism and progression; and could enable treatment response measurements for MCT-targeted cancer therapies. Moreover, it may provide vital insights into the signal kinetics of hyperpolarised [1-13C]pyruvate examinations.

\section{Acknowledgements}

This work was supported by a grant from the German Bundesministerium für Bildung und Forschung (BMBF FKZ 13EZ1114) and DFG grant SFB 824. The authors thank Annette Frank and Giaime Rancan for animal handling, as well as Geoffrey Topping for technical advice. 


\section{References}

1. Gatenby RA, Gillies RJ. Why do cancers have high aerobic glycolysis? Nat Rev Cancer 2004;4(11):891-899.

2. Kim JW, Dang CV. Cancer's molecular sweet tooth and the Warburg effect. Cancer Res 2006;66(18):8927-8930.

3. Halestrap AP, Price NT. The proton-linked monocarboxylate transporter (MCT) family: structure, function and regulation. Biochem J 1999;343 Pt 2:281-299.

4. Quennet V, Yaromina A, Zips D, Rosner A, Walenta S, Baumann M, Mueller-Klieser W. Tumor lactate content predicts for response to fractionated irradiation of human squamous cell carcinomas in nude mice. Radiother Oncol 2006;81(2):130-135. 
5. Grotius J, Dittfeld C, Huether M, Mueller-Klieser W, Baumann M, Kunz-Schughart LA. Impact of exogenous lactate on survival and radioresponse of carcinoma cells in vitro. Int J Radiat Biol 2009;85(11):989-1001.

6. Sattler UG, Meyer SS, Quennet V, Hoerner C, Knoerzer H, Fabian C, Yaromina A, Zips D, Walenta S, Baumann M, Mueller-Klieser W. Glycolytic metabolism and tumour response to fractionated irradiation. Radiother Oncol 2010;94(1):102-109.

7. Schwickert G, Walenta S, Sundfor K, Rofstad EK, Mueller-Klieser W. Correlation of high lactate levels in human cervical cancer with incidence of metastasis. Cancer Res 1995;55(21):4757-4759.

8. Walenta S, Wetterling M, Lehrke M, Schwickert G, Sundfor K, Rofstad EK, Mueller-Klieser W. High lactate levels predict likelihood of metastases, tumor recurrence, and restricted patient survival in human cervical cancers. Cancer Res 2000;60(4):916-921.

9. Brizel DM, Schroeder T, Scher RL, Walenta S, Clough RW, Dewhirst MW, Mueller-Klieser W. Elevated tumor lactate concentrations predict for an increased risk of metastases in headand-neck cancer. Int J Radiat Oncol Biol Phys 2001;51(2):349-353.

10. Goetze K, Walenta S, Ksiazkiewicz M, Kunz-Schughart LA, Mueller-Klieser W. Lactate enhances motility of tumor cells and inhibits monocyte migration and cytokine release. Int J Oncol 2011;39(2):453-463.

11. Baumann F, Leukel P, Doerfelt A, Beier CP, Dettmer K, Oefner PJ, Kastenberger M, Kreutz M, Nickl-Jockschat T, Bogdahn U, Bosserhoff AK, Hau P. Lactate promotes glioma migration by TGF-beta2-dependent regulation of matrix metalloproteinase-2. Neuro Oncol 2009;11(4):368-380.

12. Beckert S, Farrahi F, Aslam RS, Scheuenstuhl H, Konigsrainer A, Hussain MZ, Hunt TK. Lactate stimulates endothelial cell migration. Wound Repair Regen 2006;14(3):321-324.

13. Boidot R, Vegran F, Meulle A, Le Breton A, Dessy C, Sonveaux P, Lizard-Nacol S, Feron O. Regulation of monocarboxylate transporter MCT1 expression by p53 mediates inward and outward lactate fluxes in tumors. Cancer Res 2012;72(4):939-948.

14. Walenta S, Mueller-Klieser WF. Lactate: mirror and motor of tumor malignancy. Semin Radiat Oncol 2004;14(3):267-274.

15. Gottfried E, Kunz-Schughart LA, Ebner S, Mueller-Klieser W, Hoves S, Andreesen R, Mackensen A, Kreutz M. Tumor-derived lactic acid modulates dendritic cell activation and antigen expression. Blood 2006;107(5):2013-2021.

16. Fischer K, Hoffmann P, VoelkI S, Meidenbauer N, Ammer J, Edinger M, Gottfried E, Schwarz S, Rothe G, Hoves S, Renner K, Timischl B, Mackensen A, Kunz-Schughart L, Andreesen R, Krause SW, Kreutz M. Inhibitory effect of tumor cell-derived lactic acid on human T cells. Blood 2007;109(9):3812-3819.

17. Dietl K, Renner K, Dettmer K, Timischl B, Eberhart K, Dorn C, Hellerbrand C, Kastenberger M, Kunz-Schughart LA, Oefner PJ, Andreesen R, Gottfried E, Kreutz MP. Lactic acid and acidification inhibit TNF secretion and glycolysis of human monocytes. J Immunol 2010;184(3):1200-1209.

18. Hirschhaeuser F, Sattler UG, Mueller-Klieser W. Lactate: a metabolic key player in cancer. Cancer Res 2011;71(22):6921-6925.

19. Pinheiro C, Longatto-Filho A, Azevedo-Silva J, Casal M, Schmitt FC, Baltazar F. Role of monocarboxylate transporters in human cancers: state of the art. J Bioenerg Biomembr 2012;44(1):127-139.

20. Pertega-Gomes N, Baltazar F. Lactate transporters in the context of prostate cancer metabolism: what do we know? Int J Mol Sci 2014;15(10):18333-18348.

21. Baltazar F, Pinheiro C, Morais-Santos F, Azevedo-Silva J, Queiros O, Preto A, Casal M. Monocarboxylate transporters as targets and mediators in cancer therapy response. Histol Histopathol 2014;29(12):1511-1524.

22. Coss RA, Storck CW, Daskalakis C, Berd D, Wahl ML. Intracellular acidification abrogates the heat shock response and compromises survival of human melanoma cells. Mol Cancer Ther 2003;2(4):383-388. 
23. Colen CB, Seraji-Bozorgzad N, Marples B, Galloway MP, Sloan AE, Mathupala SP. Metabolic remodeling of malignant gliomas for enhanced sensitization during radiotherapy: an in vitro study. Neurosurgery 2006;59(6):1313-1323; discussion 1323-1314.

24. Mathupala SP, Colen CB, Parajuli P, Sloan AE. Lactate and malignant tumors: a therapeutic target at the end stage of glycolysis. J Bioenerg Biomembr 2007;39(1):73-77.

25. Colen CB, Shen Y, Ghoddoussi F, Yu P, Francis TB, Koch BJ, Monterey MD, Galloway MP, Sloan $A E$, Mathupala SP. Metabolic targeting of lactate efflux by malignant glioma inhibits invasiveness and induces necrosis: an in vivo study. Neoplasia 2011;13(7):620-632.

26. Ardenkjaer-Larsen JH, Fridlund B, Gram A, Hansson G, Hansson L, Lerche MH, Servin R, Thaning $\mathrm{M}$, Golman K. Increase in signal-to-noise ratio of $>10,000$ times in liquid-state NMR. Proceedings of the National Academy of Sciences of the United States of America 2003;100(18):10158-10163.

27. Golman K, Petersson JS. Metabolic imaging and other applications of hyperpolarized $13 \mathrm{C} 1$. Acad Radiol 2006;13(8):932-942.

28. Gallagher FA, Kettunen MI, Brindle KM. Biomedical applications of hyperpolarized C-13 magnetic resonance imaging. Prog Nucl Magn Reson Spectrosc 2009;55(4):285-295.

29. Hurd RE, Yen YF, Chen A, Ardenkjaer-Larsen JH. Hyperpolarized 13C metabolic imaging using dissolution dynamic nuclear polarization. Journal of magnetic resonance imaging : JMRI 2012;36(6):1314-1328.

30. Albers MJ, Bok R, Chen AP, Cunningham CH, Zierhut ML, Zhang VY, Kohler SJ, Tropp J, Hurd RE, Yen Y-F, Nelson SJ, Vigneron DB, Kurhanewicz J. Hyperpolarized 13C Lactate, Pyruvate, and Alanine: Noninvasive Biomarkers for Prostate Cancer Detection and Grading. Cancer Research 2008;68(20):8607-8615.

31. Day SE, Kettunen MI, Gallagher FA, Hu DE, Lerche M, Wolber J, Golman K, Ardenkjaer-Larsen $\mathrm{JH}$, Brindle KM. Detecting tumor response to treatment using hyperpolarized $13 \mathrm{C}$ magnetic resonance imaging and spectroscopy. Nature medicine 2007;13(11):1382-1387.

32. Golman K, in't Zandt R, Lerche M, Pehrson R, Ardenkjaer-Larsen JH. Metabolic imaging by hyperpolarized C-13 magnetic resonance imaging for in vivo tumor diagnosis. Cancer Res 2006;66(22):10855-10860.

33. Brindle KM, Bohndiek SE, Gallagher FA, Kettunen MI. Tumor imaging using hyperpolarized 13C magnetic resonance spectroscopy. Magnetic resonance in medicine : official journal of the Society of Magnetic Resonance in Medicine / Society of Magnetic Resonance in Medicine 2011;66(2):505-519.

34. Harris T, Eliyahu G, Frydman L, Degani H. Kinetics of hyperpolarized 13C1-pyruvate transport and metabolism in living human breast cancer cells. Proc Natl Acad Sci U S A 2009;106(43):18131-18136.

35. Keshari KR, Sriram R, Koelsch BL, Van Criekinge M, Wilson DM, Kurhanewicz J, Wang ZJ. Hyperpolarized 13C-pyruvate magnetic resonance reveals rapid lactate export in metastatic renal cell carcinomas. Cancer Res 2013;73(2):529-538.

36. Kettunen MI, Hu DE, Witney TH, McLaughlin R, Gallagher FA, Bohndiek SE, Day SE, Brindle KM. Magnetization transfer measurements of exchange between hyperpolarized [113C]pyruvate and [1-13C]lactate in a murine lymphoma. Magnetic resonance in medicine : official journal of the Society of Magnetic Resonance in Medicine / Society of Magnetic Resonance in Medicine 2010;63(4):872-880.

37. Hu S, Zhu M, Yoshihara HA, Wilson DM, Keshari KR, Shin P, Reed G, von Morze C, Bok R, Larson $\mathrm{PE}$, Kurhanewicz J, Vigneron DB. In vivo measurement of normal rat intracellular pyruvate and lactate levels after injection of hyperpolarized [1-(13)C]alanine. Magn Reson Imaging 2011;29(8):1035-1040.

38. Smith MR, Peterson ET, Gordon JW, Niles DJ, Rowland IJ, Kurpad KN, Fain SB. In vivo imaging and spectroscopy of dynamic metabolism using simultaneous $13 \mathrm{C}$ and $1 \mathrm{H}$ MRI. IEEE Trans Biomed Eng 2012;59(1):45-49. 
39. Kettunen MI, Kennedy BW, Hu DE, Brindle KM. Spin echo measurements of the extravasation and tumor cell uptake of hyperpolarized [1-(13) C]lactate and [1-(13) C]pyruvate. Magn Reson Med 2013;70(5):1200-1209.

40. Yen YF, Le Roux P, Mayer D, King R, Spielman D, Tropp J, Butts Pauly K, Pfefferbaum A, Vasanawala S, Hurd R. T(2) relaxation times of (13)C metabolites in a rat hepatocellular carcinoma model measured in vivo using (13)C-MRS of hyperpolarized [1-(13)C]pyruvate. NMR in biomedicine 2010;23(4):414-423.

41. Schilling F, Duwel S, Kollisch U, Durst M, Schulte RF, Glaser SJ, Haase A, Otto AM, Menzel MI. Diffusion of hyperpolarized (13) C-metabolites in tumor cell spheroids using real-time NMR spectroscopy. NMR in biomedicine 2013;26(5):557-568.

42. Sogaard LV, Schilling F, Janich MA, Menzel MI, Ardenkjaer-Larsen JH. In vivo measurement of apparent diffusion coefficients of hyperpolarized (1)(3)C-labeled metabolites. NMR Biomed 2014;27(5):561-569.

43. Adamson E, Gordon J, Johnson K, Fain S. SU-E-QI-11: Measurement of Renal Pyruvate-ToLactate Exchange with Hyperpolarized 13C MRI. Med Phys 2014;41(6):379-379.

44. Larson PE, Hurd RE, Kerr AB, Pauly JM, Bok RA, Kurhanewicz J, Vigneron DB. Perfusion and diffusion sensitive $13 \mathrm{C}$ stimulated-echo MRSI for metabolic imaging of cancer. Magn Reson Imaging 2013;31(5):635-642.

45. Koelsch BL, Reed GD, Keshari KR, Chaumeil MM, Bok R, Ronen SM, Vigneron DB, Kurhanewicz $\mathrm{J}$, Larson PE. Rapid in vivo apparent diffusion coefficient mapping of hyperpolarized $\mathrm{C}$ metabolites. Magn Reson Med 2014.

46. Terreno E, Botta M, Fedeli F, Mondino B, Milone L, Aime S. Enantioselective recognition between chiral alpha-hydroxy-carboxylates and macrocyclic heptadentate lanthanide(III) chelates. Inorg Chem 2003;42(16):4891-4897.

47. Gabellieri C, Leach MO, Eykyn TR. Modulating the relaxivity of hyperpolarized substrates with gadolinium contrast agents. Contrast Media Mol Imaging 2009;4(3):143-147.

48. Terreno E, Botta M, Boniforte P, Bracco C, Milone L, Mondino B, Uggeri F, Aime S. A multinuclear NMR relaxometry study of ternary adducts formed between heptadentate Gd(III) chelates and L-lactate. Chemistry 2005;11(19):5531-5537.

49. Derby K, Tropp J, Hawryszko C. Design and evaluation of a novel dual-tuned resonator for spectroscopic imaging. Journal of Magnetic Resonance (1969) 1990;86(3):645-651.

50. Schulte RF, Wiesinger F. Direct design of 2D RF pulses using matrix inversion. J Magn Reson 2013;235:115-120.

51. Schulte RF, Sperl JI, Weidl E, Menzel MI, Janich MA, Khegai O, Durst M, Ardenkjaer-Larsen JH, Glaser SJ, Haase A, Schwaiger M, Wiesinger F. Saturation-recovery metabolic-exchange rate imaging with hyperpolarized [1-13C] pyruvate using spectral-spatial excitation. Magnetic resonance in medicine : official journal of the Society of Magnetic Resonance in Medicine / Society of Magnetic Resonance in Medicine 2013;69(5):1209-1216.

52. Hargreaves BA. Spin-Manipulation Methods for Efficient Magnetic Resonance Imaging [Ph.D. Thesis]: Stanford University; 2001.

53. Fessler JA. On NUFFT-based gridding for non-Cartesian MRI. Journal of magnetic resonance 2007;188(2):191-195.

54. Gudbjartsson H, Patz S. The Rician distribution of noisy MRI data. Magn Reson Med 1995;34(6):910-914.

55. Weidensteiner C, Rausch M, McSheehy PM, Allegrini PR. Quantitative dynamic contrastenhanced MRI in tumor-bearing rats and mice with inversion recovery TrueFISP and two contrast agents at 4.7 T. Journal of magnetic resonance imaging : JMRI 2006;24(3):646-656.

56. Dimmer KS, Friedrich B, Lang F, Deitmer JW, Broer S. The low-affinity monocarboxylate transporter MCT4 is adapted to the export of lactate in highly glycolytic cells. The Biochemical journal 2000;350 Pt 1:219-227.

57. Ullah MS, Davies AJ, Halestrap AP. The plasma membrane lactate transporter MCT4, but not MCT1, is up-regulated by hypoxia through a HIF-1alpha-dependent mechanism. The Journal of biological chemistry 2006;281(14):9030-9037. 
58. Broer S. Adaptation of plasma membrane amino acid transport mechanisms to physiological demands. Pflugers Arch 2002;444(4):457-466.

59. Shennan DB, McNeillie SA, Curran DE. The effect of a hyposmotic shock on amino acid efflux from lactating rat mammary tissue: stimulation of taurine and glycine efflux via a pathway distinct from anion exchange and volume-activated anion channels. Exp Physiol 1994;79(5):797-808.

60. Durst M, Koellisch U, Gringeri C, Janich MA, Rancan G, Frank A, Wiesinger F, Menzel MI, Haase A, Schulte RF. Bolus tracking for improved metabolic imaging of hyperpolarised compounds. Journal of magnetic resonance 2014;243:40-46.

Tables

\begin{tabular}{|l|l|l|l|}
\hline & Pyr [5 mM] & Lac [0.5 mM] & Ala [0.5 mM] \\
\hline Gd-HP-DO3A [1 mM] & $\mathbf{2 2 0 0 \pm 3 0 0 ~ m s}$ & $500 \pm 50 \mathrm{~ms}$ & $\mathbf{7 0 0} \pm 20 \mathrm{~ms}$ \\
\hline GdDO3A [1 mM] & $90 \pm 10 \mathrm{~ms}$ & $43 \pm 5 \mathrm{~ms}$ & $35 \pm 5 \mathrm{~ms}$ \\
\hline GdDO3A [0.1 mM] & $800 \pm 50 \mathrm{~ms}$ & $400 \pm 50 \mathrm{~ms}$ & $330 \pm 30 \mathrm{~ms}$ \\
\hline
\end{tabular}

Table 1. $T_{1}$ measured in serum for pyruvate, lactate and alanine in the presence of Gd-HPDO3A and GdDO3a.

\begin{tabular}{|l|l|l|l|l|}
\hline rat & acquisition & $\begin{array}{l}\text { conc. ratio } \\
\text { Gd:Pyr }\end{array}$ & $\begin{array}{l}\boldsymbol{k}_{\mathbf{L} \text {,int } \rightarrow \text { ext }} \\
{\left[\mathbf{s}^{-1}\right]}\end{array}$ & $\begin{array}{l}\boldsymbol{k}_{\text {A,int } \rightarrow \text { ext }} \\
{\left[\mathbf{s}^{-1}\right]}\end{array}$ \\
\hline 1 & spectr. & $1: 5$ & $0.16 \pm 0.01$ & $0.02 \pm 0.01$ \\
\hline 2 & spectr & $1: 5$ & $0.19 \pm 0.04$ & $0.00 \pm 0.01$ \\
\hline 3 & spectr. & $1: 5$ & $0.25 \pm 0.04$ & $0.01 \pm 0.01$ \\
\hline 4 & spectr. & $1: 1$ & $0.31 \pm 0.07$ & $0.02 \pm 0.01$ \\
\hline 5 & imaging & $1: 5$ & $0.15 \pm 0.04$ & $0.02 \pm 0.02$ \\
\hline 6 & Imaging & $1: 5$ & $0.18 \pm 0.01$ & $0.02 \pm 0.02$ \\
\hline 7 & Imaging & $1: 5$ & $0.16 \pm 0.05$ & $0.03 \pm 0.02$ \\
\hline 8 & imaging & $1: 5$ & $0.22 \pm 0.03$ & $-0.01 \pm 0.03$ \\
\hline
\end{tabular}

Table 2. Results from in vivo experiments in Fisher F344 rats bearing a subcutaneous Mat BIII tumour in the neck region. Given are the mode of acquisition (spectroscopy or imaging), the concentration ratio of GdDO3A and the apparent cell membrane transporter activity rates (lactate: $k_{L, \text { int } \rightarrow \text { ext }}$, alanine: $k_{A, \text { int } \rightarrow \text { ext }}$ ). Errors are propagated $95 \%$ confident intervals. 


\section{Figures}

Figure 1. Formation of a reversible ternary adduct between GdDO3A and lactate (see $(46,48))$.

Figure 2. In vivo signal kinetics of hyperpolarised [1-13C]pyruvate in a cancer cell. Left: Standard NMR acquisition, intra- and extracellular compartments cannot be separated, right: saturation pulse destroys pyruvate magnetisation and only intracellular metabolite signals are left. If transported out of the cell, the metabolite magnetisation will be quenched by GdDO3A. Therefore, the MCT transporter activity manifests as additional decay in the presence of GdDO3A.

Figure 3. Acquisition schemes for the presented substrate saturation sequence. Top row: FID readout without gradient; bottom row: imaging acquisition with single-shot spiral gradient readout.

Figure 4. Representative dataset from a preliminary study without saturation pulse: Spectroscopic acquisitions of a kidney slice (slice thickness $14 \mathrm{~mm}$ ) from a healthy rat after injection of hyperpolarised [1-13C]pyruvate. The plot shows the metabolic time curves without (blue) and with administration of GdDO3A (red) for lactate (a) and alanine (b). A significant difference can be observed for both metabolites, which could be due to efflux or loss of hyperpolarised flux from pyruvate. The proposed method with saturation pulse allows resolution of this issue.

Figure 5. Representative dataset from rat 1 for spectroscopic acquisition. The plot shows metabolite decay without (blue) and with administration of GdDO3A (red) as well as the corresponding monoexponential fits (black) for lactate (a) and alanine (b). A significant difference in decay can be observed for lactate, indicating rapid lactate efflux.

Figure 6. Representative dataset from rat 6 for imaging acquisition. a) FRFSE proton image for anatomical reference b) Lactate signal (sum of first three images) overlaid with a proton image c) Apparent MCT transporter map overlayed with a proton image (signal masked for > $30 \%$ of the maximum).

Supplementary Figures 
Figure 1. Time series of DCE measurement (zoomed in at the tumour). A time resolved imaging of contrast kinetics (TRICKS) sequence was used to acquire T1-weighted proton images every $2.67 \mathrm{~s}$ (resolution $128 \times 128$ pixels, FOV $8 \mathrm{~cm}$ ).

Figure 2. $13 \mathrm{C}$ relaxation rates of the carboxylate signals of lactate (filled circles, red) and alanine (open circles, blue) as a function of the time-dependent concentration of GdDO3A in the blood flow, after the injection of CA. These values have been derived from in vitro measurements of $13 \mathrm{C}$ relaxation rates of lactate and alanine using approximately the same concentration as in blood of the metabolites and the Gd complex. The time dependent concentration of the CA has been derived from DCE study.

Figure 3. MCT1 immunohistochemical assay (Merck-Millipore AB1286-I), bars $50 \mu \mathrm{m}$.

Figure 4. MCT4 immunohistochemical assay (Santa Cruz Biotechnologies sc-50329), bars 50 $\mu \mathrm{m}$. 


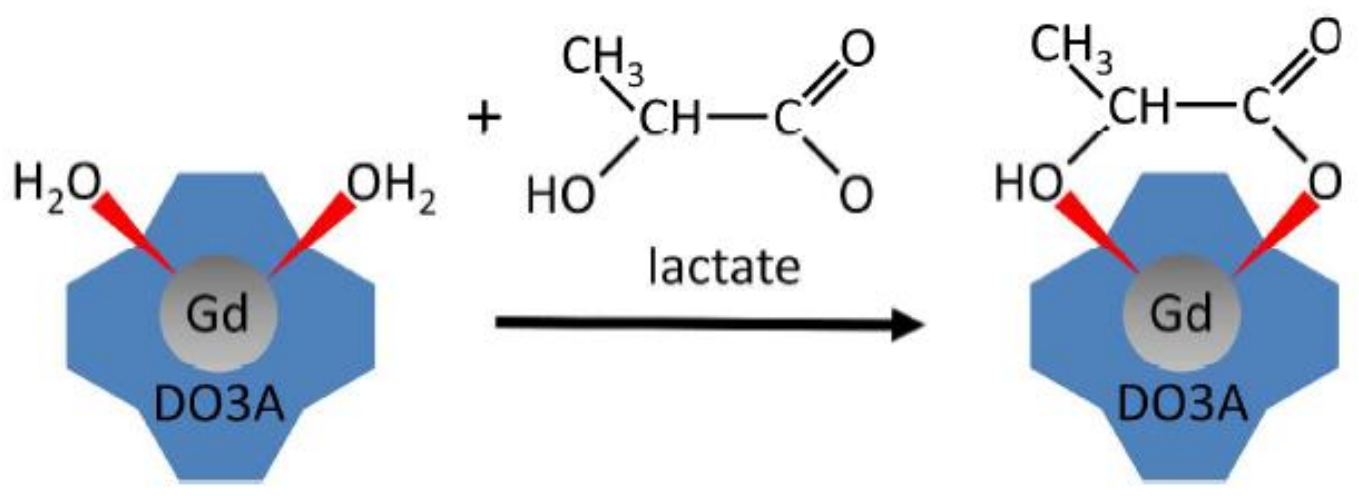

Figure 1

In vivo signal kinetics of hyperpolarised [1-13C]pyruvate

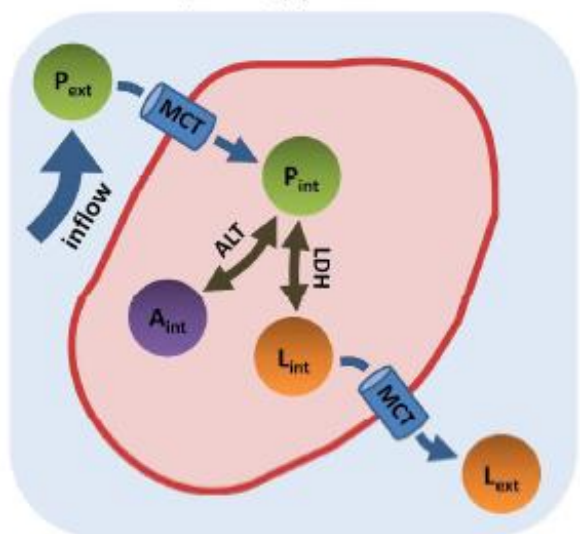

In vivo signal kinetics of hyperpolarised [1-13C]pyruvate + GdDO3A + satpulse

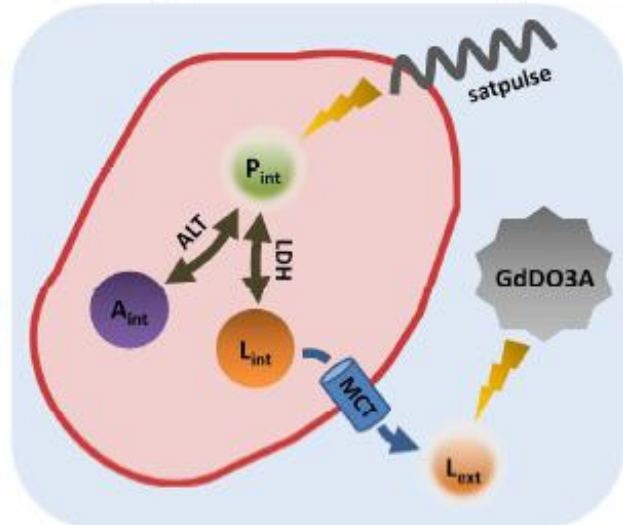

Figure 2 


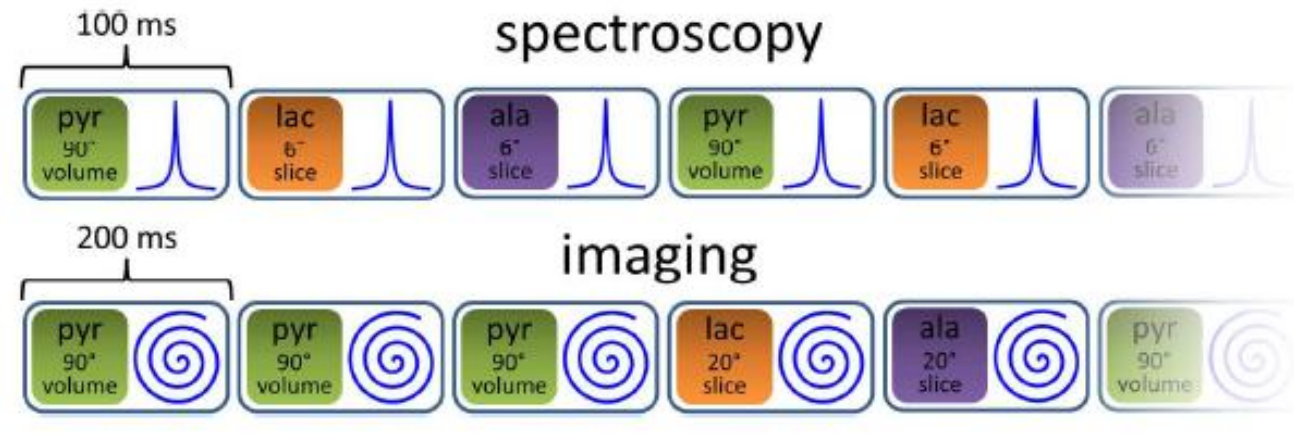

Figure 3.
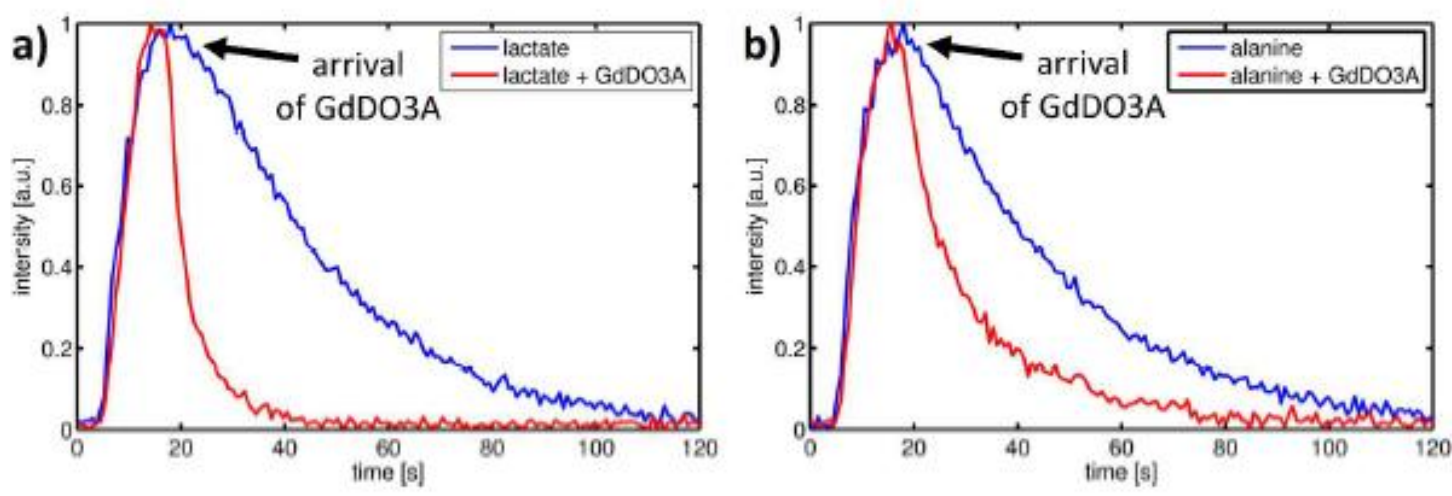

Figure 4.
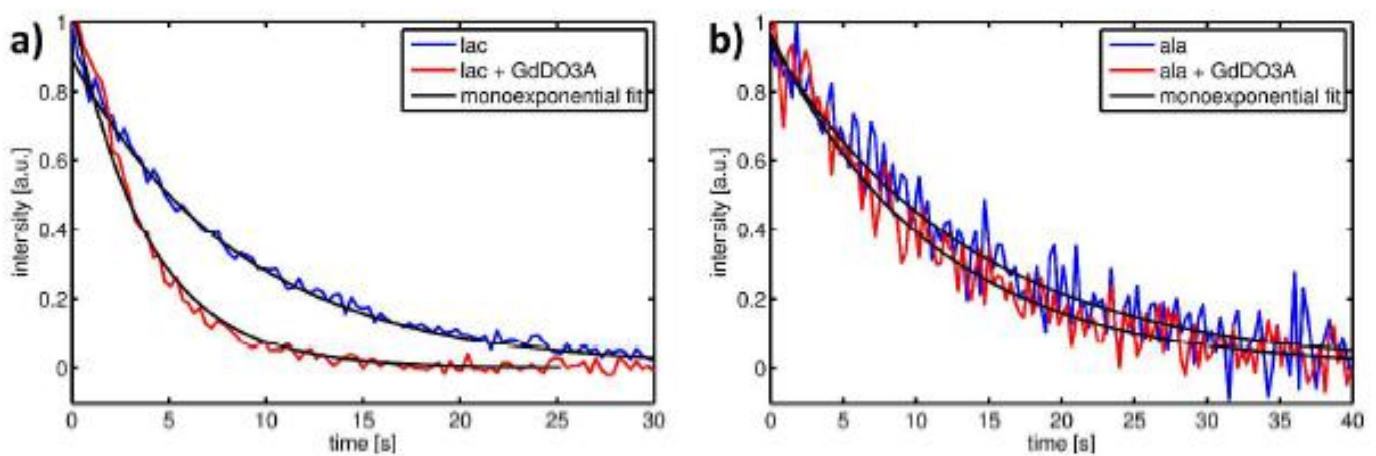

Figure 5. 


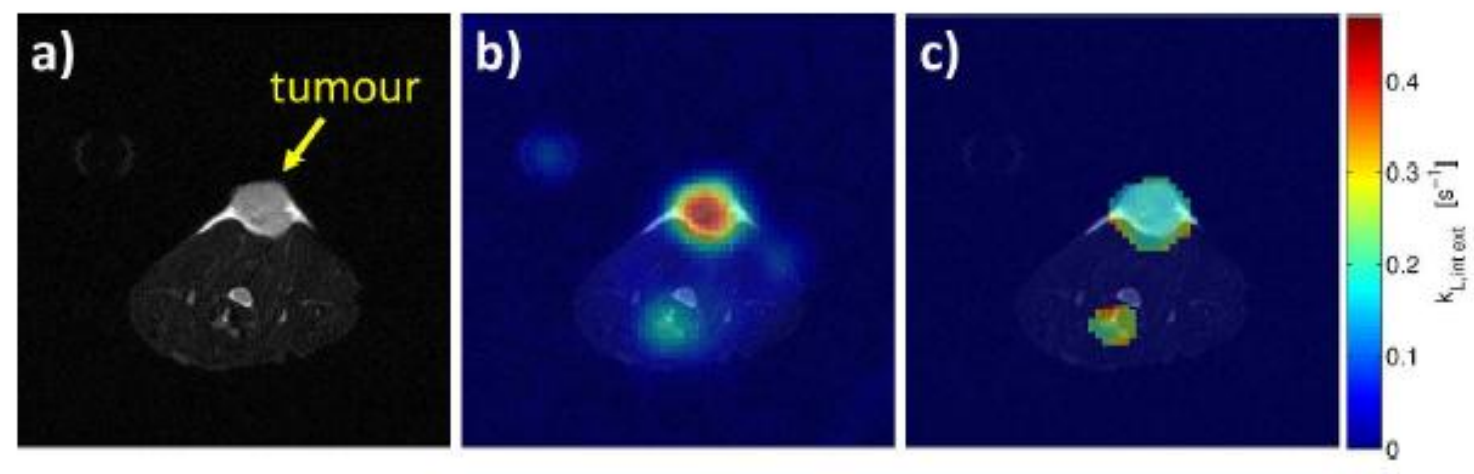

Figure 6. 